\title{
The Trajectory Dependent Scattering of Li Ions by Al Substrate
}

\author{
V. KRÁL \\ Dolná 12, Prague 4, 14 000, Czech Republic
}

(Received July 7, 2009)

\begin{abstract}
The trajectory dependent scattering of Li ions by $\mathrm{Al}$ substrate is studied. It is shown that besides primary elastic peak there exists a subsidiary peak about one-third energy of incident ions. The dependence of trajectory is achieved by shifting of the incident beam along $x$ axis. The results have been compared with the results of Na scattering that have been published recently.
\end{abstract}

PACS numbers: 79.20.Rf

\section{Introduction}

Scattering of ions by solids is a powerful tool for studying of solid surfaces. Many techniques like secondary ion mass spectroscopy (SIMS) have been developed and they give at present the convincing information about solid surfaces. The purpose of this contribution is to give a description of trajectory dependent scattering of $\mathrm{Li}$ ions by $\mathrm{Al}$ surface. The trajectory dependent scattering has been reported in [1] for incident $\mathrm{Na}$ ions. They show that results of scattering depend on the place of surface by which is a ion scattered. The spectra obtained in these experiments allow to recognize the origin of outgoing ions. One can imagine this situation by a following reasoning.

\section{Results and discussion}

In Fig. 1 one can see the scattering of ions by upper layer and lower layer, respectively. Scattering of ions may be described by shielded Coulomb potential, $V=\mathrm{e}^{-\mu r} / r$. Since the scattering properties of upper and lower layer are different, the cross-section differs for upper and lower layer. One may estimate this difference by formula [2]:

$$
\mathrm{d} \sigma^{\prime} / \mathrm{d} \Omega=\left[1 /\left(4 k^{2} \sin ^{2}(\theta / 2)+\mu^{2}\right)\right]^{2},
$$

which reflects the dependence on a scattering potential.

As we mentioned above, the trajectory dependent scattering has been reported for system $\mathrm{Na}-\mathrm{Cu}$ in [1].

The results show in the outgoing spectra a peak corresponding to process (a) in Fig. 1 and the subsidiary peak which corresponds to process (b) in Fig. 1.

Energy of the outgoing ions has been measured in perpendicular velocity in atomic units. The energy of incident $\mathrm{Na}$ ions in [1] has been used as $50 \mathrm{eV}$ and the angle of the incident beam has been $3^{\circ}$ while the outgoing ions

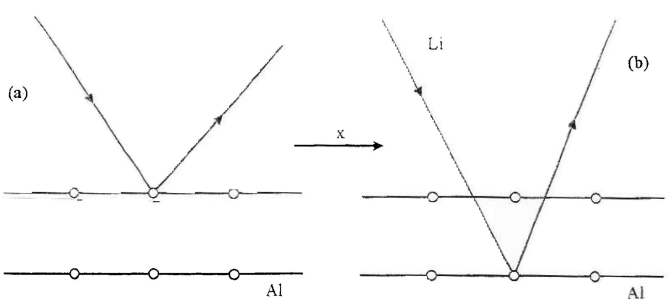

Fig. 1. Scattering of $\mathrm{Li}$ ions by $\mathrm{Al}$ surface by upper layer (a) and by lower layer (b).

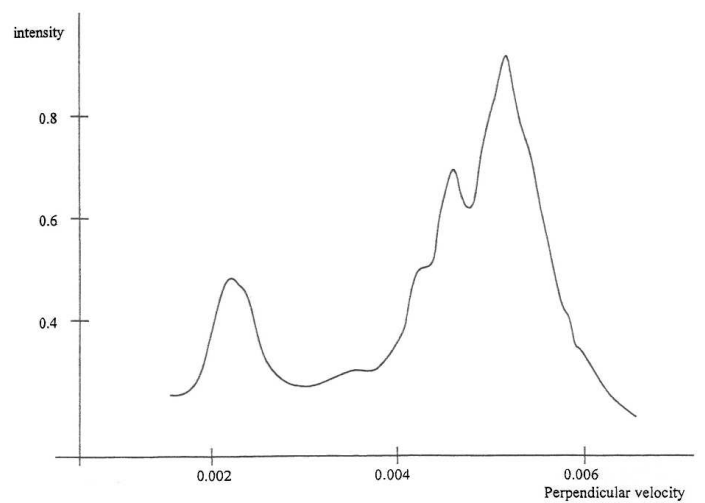

Fig. 2. Scattering of $\mathrm{Na}$ ions by $\mathrm{Cu}$ according to [1].

were scattered at $35^{\circ}$. We reproduce the spectra obtained in [1] schematica II in Fig. 2. Atomic unit in velocity is equal to $2.2 \times 10^{-8} \mathrm{~cm} \mathrm{~s}^{-1}$.

In our contribution we have studied the scattering of $\mathrm{Li}$ ions by $\mathrm{Al}$ surface. The energy of incident ions has been $1050 \mathrm{eV}$. The potential due to the scattering took place has been chosen either as Moliere one [3], or the 
embedded atom potential (EAP) [4, 5], instead of BornMayer type of Hartree-Fock potential used in [1]. Instead of SAFARI code [6], used in [1], we have used the code based on the Runge-Kutta solution of equations of motion [3]:

$$
m \mathrm{~d}^{2} x / \mathrm{d} t^{2}=-\sum_{i j} \operatorname{grad} V_{i j} .
$$

We did not consider the charge exchange which was studied in [1], neither we studied the forming of neutrals in outgoing beam. The charge exchange is studied in detail in [7].

The scattering of Li ions by $\mathrm{Al}$ substrate has been studied in [8]. The spectra obtained in [8] may be estimated by the following formula which is based on the theory of binary collisions

$$
\begin{gathered}
E_{1}=E_{0}\left[\left(\cos \Psi+\sqrt{m_{1}^{2} / m_{2}^{2}}-\sin ^{2} \psi\right)\right. \\
\left./\left(1+m_{2} / m_{1}\right)\right]^{2} .
\end{gathered}
$$

The outgoing Li ions form two peaks, one is almost elastic one while the subsidiary peak occurs in lower energy. In our study of the trajectory dependent scattering of $\mathrm{Li}$ ions by $\mathrm{Al}$ surface we have found in accordance with [1] two peaks. The main peak is almost elastic one and its origin is due to process depicted in Fig. 3 as the scattering by upper layer. The other peak of the energy approximately one-third of primary peak is due to the scattering by lower layer, process depicted in Fig. 3. So the spectra of outgoing particles allow us to understand the origin of the scattered ions. The dependence on the trajectory of particles is in our modelling achieved by shifting the incident beam by $x$ axis.

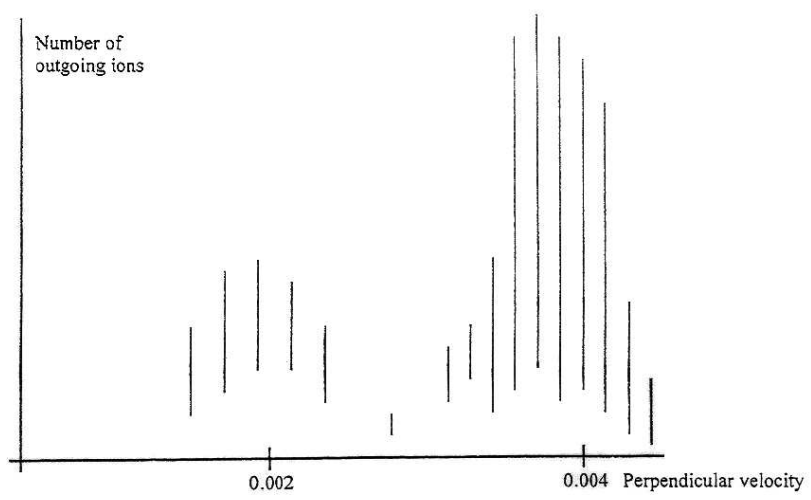

Fig. 3. Spectra of outgoing $\mathrm{Li}$ ions scattered by $\mathrm{Al}$ surface.

\section{Conclusion}

Our spectra show the similar features to those reported in [1] although the potentials used in our simulations were Moliere or EAP potential instead of Born-Mayer potential used in [1]. The results seem to be rather insensitive to a choice of potential. The simulation in our study was based on the Runge-Kutta solution of the equations of motion while in [1] SAFARI code was used. The problem of charge exchange and forming the neutrals has not been solved in our contribution.

\section{References}

[1] C.A. Keller, C.A. diRubbio, G.A. Kimmel, B.H. Cooper, Phys. Rev. Lett. 75, 1654 (1995).

[2] A.S. Davydov, Quantum Mechanics, SPN, Prague 1978.

[3] V. Král, Czech. J. Phys. 55, 497 (2005).

[4] M.S. Daw, M.I. Baskes, Phys. Rev. B 29, 6443 (1984).

[5] S.M. Foiles, M.I. Baskes, M.S. Daw, Phys. Rev. B 33, 7688 (1986).

[6] D.M. Goodstein, S.A. Langer, B.H. Cooper, J. Vac. Sci. Technol. A 6, 703 (1988).

[7] J. Los, J.J.C. Gerlings, Phys. Rep. 190, 134 (1990).

[8] K.A.H. German, G.B. Weare, J.A. Yarmoff, Phys. Rev. B 50, 14452 (1994). 